\title{
Vibration Analysis for Engine Fault Detection
}

\author{
Angad Gude ${ }^{1}$, Shubham Pawar $^{2}$, Siddharth Alhat ${ }^{3}$, Sashikala Mishra ${ }^{4}$ \\ Department of Computer Engineering, International Institute of Information Technology, Pune
}

\begin{abstract}
In the Vibration analysis for engine fault detection, we use different visualization graph. Today's world growing fast and machinery part getting complex so it's difficult to find out fault in the machine so here means in this paper we explain how we find out the fault of the machine with help of visualization it's easy to find out a fault here we use angular.js, D3.js for visualization and use MQTT protocol for publishing and subscribe sensor data. In the automobile industries machines are the main part of how we find out fault yes we find out fault with help of sensors using sensors here we analyze the machine.
\end{abstract}

Keywords - Sensor, Protocol, Visualization, Fault detection, Graph

\section{INTRODUCTION}

Automobile Science is progressing increasing with an astonishing speed and car manufacturing is competing with each other to reach more targets in markets. But to achieve target company manufacturing the product rapidly [1]. Because of the rapid production the machine may get some defect to it. It may be not visible to the eye, but that may result into serious accident to avoid such accident we are measuring the VIBRATION of the machine [2]-[5]. Through vibration analysis of the machine/Engine behavior it's possible to screen the problematic or the doubtful machine [6]-[10]. There are different type of engine fault [11]-[18] like spark plug gap, valve clearance (inlet and outlet), air filter fault, piston ring fault, inlet and exhaust manifold fault and injector valve [19]. All machines with moving parts give rise to sound and vibration. Each machine has a specific vibration signature related to the construction and the state of the machine. If the state of the machine changes the vibration signature will also change. Most modern techniques for diesel engine diagnostics are recognized on the analysis of vibration signals collected from cylinder block or the head. A change in the vibration signature can be used to detect initial faults before they become critical [20]-[39]. The data flow from the sensor to the dashboard is described by the data flow diagram shown in Figure 1.

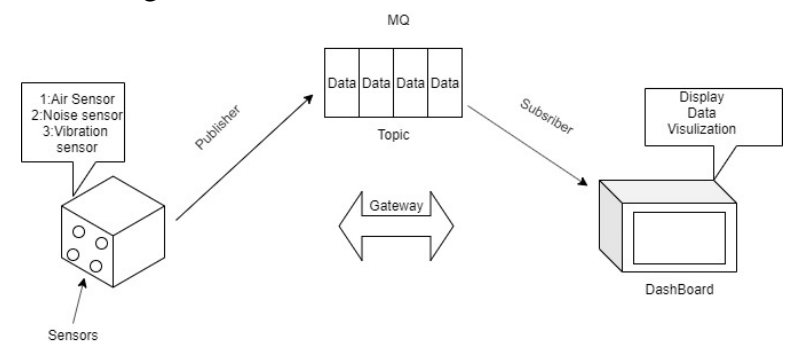

Fig 1. data flow diagram
From Figure 1, it can be seen that the system consists of sensors, protocol and dashboard. Sensor is used for engine fault detection we need sensor [31]-[34] for identify the fault in engine so there are different sensor which is list out below so basically this sensor sense the engine data like vibration data, Noise data and provide this data for fault detection there are some protocol we use here for getting data from sensor .This sensor data transfer from MQTT to using publisher and subscriber script.

- Vibration Sensor

- Noise Sensor

- Air Sensor

MQTT protocol is nothing but messing queue telemetry transport its work as gateway means its work as interface between sensor and dashboard mqtt as also known as broker have publisher and subscriber like sensor sense data of machine and then mqtt get this data and store it in database after subscriber put data on dashboard. This work as middle ware because sensor data collect in topic different topic like. Dashboard basically Graphical User Interface for user. Dashboard is nothing but output window mean what user want they can request like if they want to show graphs there are different graphs like line, bar, pie chart etc.

\section{LITERATURE REVIEW}

Kamal Jafarian, Morteza Darjani, Zahra Honarkar proposed to detect and categorize faults appeared due to poppet valve clearance and incomplete combustion sometimes called misfiring phenomenon in internal combustion engine [19]. So we used four accelerometers on OHV engine body for registering vibration signals. Then using PCA technique acquiring data were investigated. At the end we succeeded to classify and detect faults with a high ratio of efficiency.

Pal Banlaki, Zoltan Magosi proposed to develop a flexible and improvable measurement system which is usable both for experiments and final tests of several engines [30]. Using the measuringset-upwecouldexploit the order analysis function together with the analysis of results in the frequency domain.

Sabry Allam, Mohammed Abdo and Dr. M. Rabie proposed The vibration and acoustic methods are among the most useful techniques for monitoring the conditions of machines [1]. The internal combustion engine one of machine which has the reciprocating parts can cause progressive damage that ultimately affects the engine efficiency.

Himanshu K. Patel, Dhagash Shah, Avani Raghuwanshi proposed in This paper overviews the generalized health monitoring concept for machines and presents the health monitoring of a rotating machine based on Vibration Data Analysis using an enhanced Fast Fourier Transform Approach [35]. 


\section{ARCHITECTURE DIAGRAM}

The architecture diagram of the system is shown in Figure 2. From this figure, it can be seen that the system consists of a first layer, a second layer and a third layer.

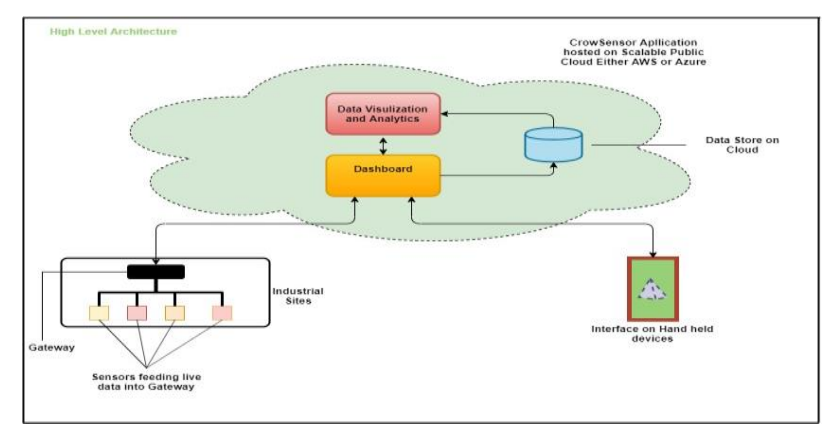

Fig 2. Architecture diagram

- First Layer: In the first layer there are different types of sensors like vibration, sound sensor etc. This sensor is connected to engine to find out the fault this sensor reading data of engine, here we use gateway to accessing data from sensor using MQTT protocol mqtt is work as messaging queue where we get sensor data as topic format publisher and subscriber are play important role here publisher publish topic data and then subscriber subscribe data depend on topic like what user want which topic they want vibration, sound topic.

- Second Layer: In the second layer Visualization part comes under where visualize sensor data using D3.js and Angular.js technology. Visualize data on dashboard Visualization is nothing but graphical representation of data, data visualize in different graph like Bar, Line and scatter plot. It is providing the visualization of data through the different types of graphs it needs to be representing it on any platform. So as shown in the diagram dashboard is provided for it. In dashboard user will able to login and can see it.

- Third Layer: This is last layer this layer comes under in storing data showing how to collect data through sensor and store data on cloud and also store visualization data on cloud. After getting sensor data store this data on cloud. Cloud providing different services like accessing data where user can access data easily.

\section{WORKING PROCEDURE}

Data Collection: First the sensors will be connected to the machine so it can generate the data. After that the data is send through the mqtt queue through the publisher. Publisher will be publishing the data to the mqtt queue and mqtt will hold the data and send it to the subscriber who will subscribe through the topic name.

Example: Consider these duplicate data.

- Input from the sensor to the mqtt
[Publishing]Topic=STVOM-4f7788

Message $=\{$ "Gx":-11,"Gy":197,"Gz":-

150,"ThetaX":32,"ThetaY":-65,"Temp":27\}

- $\quad$ Output from the mqtt to the subscriber through topic name

$$
\begin{aligned}
& \text { Topic=STVOM-4f7788 Message= } \\
& \{\text { "Gx":103,"Gy":-193,"Gz":- } \\
& \text { 67,"ThetaX":2,"ThetaY":88,"Temp":31\} }
\end{aligned}
$$

Data visualization: In data visualization the data is taken from the sensor through the mqtt queue and visualized in the form of graph like line graph, bar graph, and scatter plot. To plot this graph, we used the $\mathrm{d} 3$ library and angular framework.

Data storing: The data will be store on the cloud database. But all the data will be not stored on the cloud. The data is crossing the threshold values only that data will be store on the cloud database.

\section{OUTPUT}

In Figure 3 is Line Graph which is representing flow of sensor data. Here we get the data from sensor in numeric form and represent this data in line graph where we can easily understand the fault of machine.

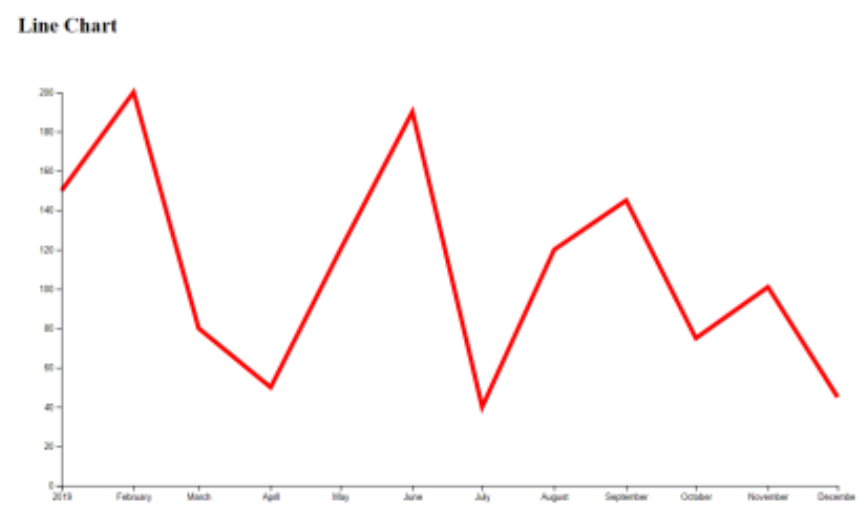

Fig 3. Line Graph

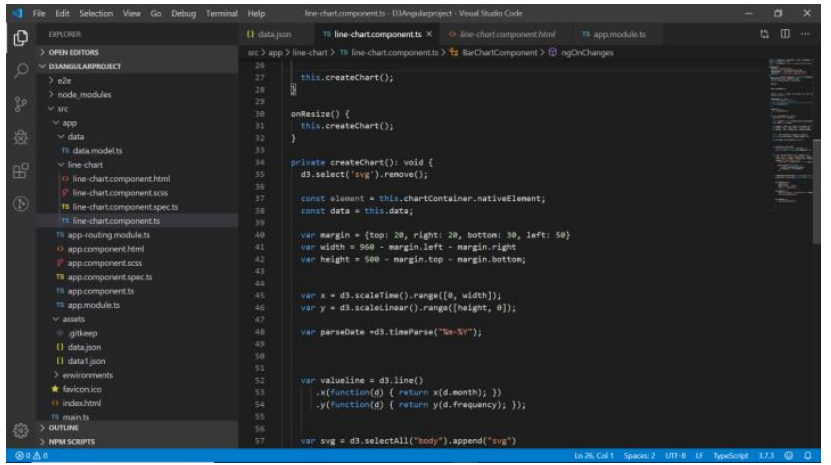

Fig 4. Implimentation

This is implementation part which is represent backend where we use angular js for visualizing the sensor data. 


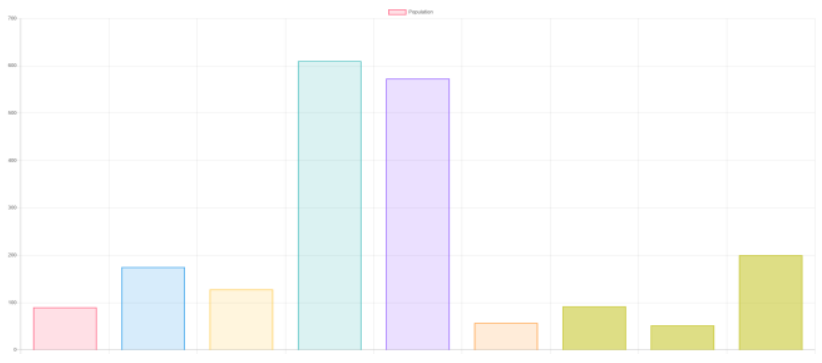

Fig. 5. Bar graph

As shown fig 5 outputs of sensors in bar graph and on Dashboard there are different types of graphs like bar, line, scatter etc. In this fig3.4 X-axis showing multiple sensors like vibration, Noise, Temperature, Air with help of bar on X-axis and $\mathrm{Y}$-axis showing the range of sensor and range is numeric form this is how we can analysis the sensors data by specific period of time (e.g. by day, by moth) with help of bar graph this graph represented with help of D3.js and Angular.js.

\section{CONCLUSION}

The work done in this paper allows computing the different value of the machine to identify the fault if any. This will help in the risk management to the industry and to prevent the accident. Using the feature rather than checking the machine manually one can check it without stopping the machine on the computer monitor and if will predict the condition of the machine.

\section{REFERENCES}

[1] Prof. Dr. Sabry Allam, Mohammed Abdo and Dr. M. Rabie,” Diese Engine Fault Detection Using Vibration and Acoustic Emission Signals", IJASRE-International Journal of Advances in Scientific Research and Engineering, Volume 4, Issue 12 December - 2018

[2] D. Ding, D. Zhao, X. Zhang, X. Lan, C. Li, and B. Cui, "Investigation of vibration impacts on HVAC transformer from HVDC system under monopole operation," IEEE Trans. Dielectr. Electr. Insul., vol. 23, no. 3, pp. 1386-1392, 2016.

[3] T. Li, C. Shi, Y. Tan, and Z. Zhou, "Fiber Bragg Grating SensingBased Online Torque Detection on Coupled Bending and Torsional Vibration of Rotating Shaft," IEEE Sens. J., vol. 17, no. 7, pp. 11992007, 2017.

[4] Y. Wang et al., "Optical Fiber Vibration Sensor Using Chaotic Laser," IEEE Photonics Technol. Lett., vol. 29, no. 16, pp. 1336-1339, 2017

[5] Y. Park, M. Jeong, S. Bin Lee, J. A. Antonino-Daviu, and M. Teska, "Influence of Blade Pass Frequency Vibrations on MCSA-Based Rotor Fault Detection of Induction Motors," IEEE Trans. Ind. Appl., vol. 53, no. 3, pp. 2049-2058, 2017.

[6] B. Liu, H. Ma, and P. Ju, "Partial discharge diagnosis by simultaneous observation of discharge pulses and vibration signal," IEEE Trans. Dielectr. Electr. Insul., vol. 24, no. 1, pp. 288-295, 2017.

[7] M. Bentoumi, D. Chikouche, A. Mezache, and H. Bakhti, "Wavelet DT method for water leak-detection using a vibration sensor: An experimental analysis," IET Signal Process., vol. 11, no. 4, pp. 396405, 2017.

[8] Y. Wang and J. Pan, "Comparison of Mechanically and Electrically Excited Vibration Frequency Responses of a Small Distribution Transformer," IEEE Trans. Power Deliv., vol. 32, no. 3, pp. 1173 $1180,2017$.

[9] S. Yang, W. Wu, S. Xu, Y. J. Zhang, D. Stutts, and D. J. Pommerenke, "A Passive Intermodulation Source Identification Measurement System Using a Vibration Modulation Method," IEEE Trans. Electromagn. Compat., vol. 59, no. 6, pp. 1677-1684, 2017.

[10] Santos, R. Santos, M. Silva, E. Figueiredo, C. Sales, and J. C. W. A Costa, "A Global Expectation-Maximization Approach Based on Memetic Algorithm for Vibration-Based Structural Damage Detection,” IEEE Trans. Instrum. Meas., vol. 66, no. 4, pp. 661-670, 2017.
[11] B.-G. Gu, "Study of IPMSM Interturn Faults Part II: Online Fault Parameter Estimation," IEEE Trans. Power Electron., vol. 31, no. 10, pp. 7214-7223, Oct. 2016

[12] Tabbache, M. E. H. Benbouzid, A. Kheloui, and J.-M. Bourgeot, "Virtual-Sensor-Based Maximum-Likelihood Voting Approach for Fault-Tolerant Control of Electric Vehicle Powertrains," IEEE Trans. Veh. Technol., vol. 62, no. 3, pp. 1075-1083, Mar. 2013.

[13] Elez, S. Car, S. Tvoric, and B. Vaseghi, "Rotor Cage and Winding Fault Detection Based on Machine Differential Magnetic Field Measurement (DMFM)," IEEE Trans. Ind. Appl., vol. 53, no. 3, pp. 3156-3163, May 2017

[14] Xu, L. Sun, L. Xu, and G. Xu, "Improvement of the Hilbert Method via ESPRIT for Detecting Rotor Fault in Induction Motors at Low Slip,” IEEE Trans. Energy Convers., vol. 28, no. 1, pp. 225-233, Mar. 2013.

[15] H. Liu, J. Huang, Z. Hou, J. Yang, and M. Ye, "Stator inter-turn fault detection in closed-loop controlled drive based on switching sideband harmonics in CMV," IET Electr. Power Appl., vol. 11, no. 2, pp. 178186, Feb. 2017

[16] Soualhi, G. Clerc, and H. Razik, "Detection and Diagnosis of Faults in Induction Motor Using an Improved Artificial Ant Clustering Technique," IEEE Trans. Ind. Electron., vol. 60, no. 9, pp. 4053-4062, Sep. 2013.

[17] R. de Jesus Romero-Troncoso, "Multirate Signal Processing to Improve FFT-Based Analysis for Detecting Faults in Induction Motors," IEEE Trans. Ind. Informatics, vol. 13, no. 3, pp. 1291-1300, Jun. 2017.

[18] S. Mohanty, K. K. Gupta, and K. S. Raju, "Effect of unitary sample shifted Laplacian and rectangular distributions in bearing fault identifications of induction motor," IET Sci. Meas. Technol., vol. 11, no. 4, pp. 516-524, Jul. 2017.

[19] Kamal Jafarian, Morteza Darjani, Zahra Honarkar," Vibration Analysis for Fault Detection of Automobile Engine Using PCA Technique", 2016 4th International Conference on Control, Instrumentation, and Automation (ICCIA) 27-28 January 2016, Qazvin Islamic Azad University, Qazvin, Iran

[20] Y. Wang, B. Jin, Y. Wang, D. Wang, X. Liu, and Q. Bai, "Real-Time Distributed Vibration Monitoring System Using IPhi-OTDR," IEEE Sens. J., vol. 17, no. 5, pp. 1333-1341, 2017.

[21] Wylomanska, R. Zimroz, J. Janczura, and J. Obuchowski, "Impulsive Noise Cancellation Method for Copper Ore Crusher Vibration Signals Enhancement," IEEE Trans. Ind. Electron., vol. 63, no. 9, pp. 56125621, 2016.

[22] H. Sun, S. Yuan, and Y. Luo, "Cyclic Spectral Analysis of Vibration Signals for Centrifugal Pump Fault Characterization,” IEEE Sens. J., vol. 18, no. 7, pp. 2925-2933, 2018.

[23] T. J. Matarazzo et al., "Crowdsensing Framework for Monitoring Bridge Vibrations Using Moving Smartphones,” Proc. IEEE, vol. 106, no. 4, pp. 577-593, 2018.

[24] L. Song, H. Wang, and P. Chen, "Vibration-Based Intelligent Fault Diagnosis for Roller Bearings in Low-Speed Rotating Machinery," IEEE Trans. Instrum. Meas., vol. 67, no. 8, pp. 1887-1899, 2018.

[25] J. Wang et al., "An FBG-based 2-D vibration sensor with adjustable sensitivity,” IEEE Sens. J., vol. 17, no. 15, pp. 4716-4724, 2017.

[26] T. Li, C. Shi, Y. Tan, R. Li, Z. Zhou, and H. Ren, “A Diaphragm Type Fiber Bragg Grating Vibration Sensor Based on Transverse Property of Optical Fiber with Temperature Compensation,” IEEE Sens. J., vol. 17, no. 4, pp. 1021-1029, 2017.

[27] V. Maiwald, M. Müller, C. Ritz, C. Hierold, and C. Roman, "Offresonant vibration amplifier with flattened band-pass characteristic and improved axis selectivity," J. Microelectromechanical Syst., vol. 26, no. 6, pp. 1345-1355, 2017.J. C. Jauregui, J. R. Resendiz, S. Thenozhi, T. Szalay, A. Jacso, and M. Takacs, "Frequency and TimeFrequency Analysis of Cutting Force and Vibration Signals for Tool Condition Monitoring," IEEE Access, vol. 6, pp. 6400-6410, 2018.

[28] J. Rivas, R. Wunderlich, and S. J. Heinen, "Road Vibrations as a Source to Detect the Presence and Speed of Vehicles," IEEE Sens. J., vol. 17 , no. 2 , pp. 377-385, 2017.

[29] Pal Banlaki, Zoltan Magosi," Part failure diagnosis for internal combustion engine using noise and vibration analysis", $\begin{array}{lllll}\text { Transportation } & \text { Engineering } & \text { (2010) } & \text { 53-60 doi: }\end{array}$ 10.3311/pp.tr.2010-1.09

[30] F. R. Salmasi, "A Self-Healing Induction Motor Drive With Model Free Sensor Tampering and Sensor Fault Detection, Isolation, and Compensation," IEEE Trans. Ind. Electron., vol. 64, no. 8, pp. 61056115, Aug. 2017. 
[31] Y. C. Soh, S. K. Kommuri, J. J. Rath, M. Defoort, and K. C. Veluvolu, "Decoupled current control and sensor fault detection with secondorder sliding mode for induction motor," IET Control Theory Appl., vol. 9, no. 4, pp. 608-617, Feb. 2015.

[32] M. Manohar and S. Das, "Current Sensor Fault-Tolerant Control for Direct Torque Control of Induction Motor Drive Using Flux-Linkage Observer," IEEE Trans. Ind. Informatics, vol. 13, no. 6, pp. 28242833, Dec. 2017.

[33] Chakraborty and V. Verma, "Speed and Current Sensor Fault Detection and Isolation Technique for Induction Motor Drive Using Axes Transformation,” IEEE Trans. Ind. Electron., vol. 62, no. 3, pp. 1943-1954, Mar. 2015.

[34] Sumit Kumar Sar, Dr. Ramesh Kumar," Techniques of Vibration Signature Analysis", International Journal of Advanced Research in Computer and Communication Engineering Vol. 4, Issue 3, March 2015

[35] Priyanka, Neelam Turk, Ratna Dahiya," Condition monitoring of Induction motors through Simulation of Bearing Fault and Air Gap Eccentricity Fault", International Journal of Recent Technology and Engineering (IJRTE) ISSN: 2277-3878, Volume-8 Issue-3, September 2019

[36] Amandeep Sharma, S. Chatterji, Lini Mathew, Mohammad Junaid Khan," A Review of Fault Diagnostic and Monitoring Schemes of Induction Motors", International Journal for Research in Applied
Science \& Engineering Technology (IJRASET) Volume 3 Issue IV, April 2015

[37] Tejas K. Rathod, Prof. V.P. Patel," Research Trends in Fault Detection and Analysis of Three Phase Induction Machine", International Journal of Science, Engineering and Technology Research (IJSETR) Volume 6, Issue 3, March 2017

[38] Prof. P. C. Latane, Punit C. Urolgin," Fault Detection in Electric Motors Using Vibration Analysis and DSP Processor", International Journal of Innovative Research in Science, Engineering and Technology Vol. 5, Issue 6, June 2016 